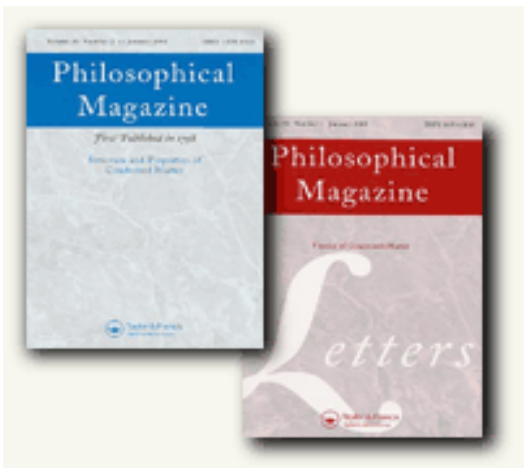

\title{
Long-range spatial correlations and scaling in dislocation and slip patterns
}

\begin{tabular}{|r|l|}
\hline Journal: & Philosophical Magazine \& Philosophical Magazine Letters \\
\hline Manuscript ID: & TPHM-06-Feb-0036.R1 \\
\hline Journal Selection: & Philosophical Magazine \\
\hline Date Submitted by the \\
Author: & 21 -Jun-2006 \\
\hline Complete List of Authors: & $\begin{array}{l}\text { Weiss, Jérôme; CNRS, LGGE } \\
\text { Montagnat, Maurine; CNRS, LGGE }\end{array}$ \\
\hline Keywords: & dislocation interactions, dislocations \\
\hline Keywords (user supplied): & scale invariance, strain gradient plasticity, dislocation density \\
\hline &
\end{tabular}

\section{s scholarONE" \\ Manuscript Central}


Long-range spatial correlations and scaling in dislocation and slip patterns

\author{
Jérôme WEISS and Maurine MONTAGNAT \\ LGGE, CNRS \\ 54 rue Molière, BP 96 \\ 38402 St Martin d'Hères Cedex \\ France
}

\begin{abstract}
Although the intermittent and heterogeneous nature of plastic flow has been known for several decades, only recently did observations performed on the surface of deformed samples by AFM or scanning white-light interferometry, or in the bulk by X-Ray topography, reveal the scale invariant character of dislocation and slip patterns emerging from collective dislocation interactions. This scale invariance implies that the spatial fluctuations of dislocation density and/or slip never vanish as one coarsens the observation scale. An immediate consequence is that a priori obvious concepts such as "slip bands" or dislocation density can be ill-defined. These detailed characterizations of the plastic flow heterogeneity also challenge the modelling of plasticity.
\end{abstract}

\title{
Keywords
}

Dislocation, dislocation density, slip line, scale invariance, long-range correlations, strain gradient plasticity

\section{Introduction}

Since the early days of the dislocation-related theory of plasticity $[1,2]$, the main question was to relate the microscale physics (from the crystal lattice scale, $b$, to the average distance between neighbouring dislocations, $l_{d}=1 / \rho^{1 / 2}$ ) to the macroscale behaviour. The celebrated Orowan's relation, $d \gamma / d t=\rho_{m}\langle v\rangle b$ which expresses the macroscopic strain-rate as a function of the density of mobile dislocations $\rho_{m}$ and of their averaged velocity $\langle v\rangle$, is based on two basic micro-macro concepts : (i) the mechanistic approach and (ii) mean-field and averaging procedures. E.g., in the Orowan's relation, it is implicitly assumed that averaging is meaningful for both $v$ and the spatial distribution of dislocations. This coarse-graining can fail 
for three reasons: (i) spatial long-range correlations exist for $\rho_{m}$, (ii) long-range correlations exist for $v$, or (iii) the distribution of $v$ is "wild", i.e. such that a variance cannot be defined and the central limit theorem cannot be applied (e.g. [3]). In other words, spatial and temporal fluctuations on $v$ and $\rho_{m}$ are too strong (more precisely, these fluctuations do not vanish relatively to the mean as the observation scale is coarsened). Recent works [4] showed that condition (iii) is often fulfilled (see below), whereas we show in this paper that conditions (i) and (ii) can be also encountered, thus questioning the applicability of such simple coarsegraining procedures in plasticity.

Since these seminal works[1,2], the notion of dislocation density has been extensively used in the literature. A well-known example is the Taylor's relation for the flow stress [5], $\sigma_{c}$ $\sim G b \rho^{1 / 2}$, which expresses, through a mean-field approach, the idea that the flow stress $\sigma_{c}$ is governed by dislocation interactions. Phenomenological models formulated in terms of differential equations for the evolution of dislocation densities and using the Taylor's relation were developed from the late 70's to model work-hardening (e.g. [6]). As stressed by Zaiser and Seeger [7], those early phenomenological density-based models were unable to account for a rather ubiquitous (at least in the case of materials with high dislocation mobility) experimental fact that became apparent at the same time: the spontaneous emergence of strong heterogeneities in the dislocation patterns.

Indeed, the intrinsic heterogeneous nature of slip, which emerge from the collective behaviour of moving dislocations, is now well-established, with dislocations tending to move cooperatively in groups of spatially correlated slip planes rather than individually and independently from each other [8]. In the spatial domain, this has been formulated by the concept of "slip lines" or "slip bands" revealed by a topographic analysis of the surface of deformed samples, a subject reviewed by Neuhauser [8] almost 25 years ago. This spatial heterogeneity is accompanied by intermittency, as plastic deformation takes place through localised "bursts" or avalanches, characterized by very large instantaneous strain-rates, whereas dislocations are almost immobile everywhere else [4]. Recent works showed that this collective dislocation dynamics is characterized by different scaling laws as well as space [9] and time [10] correlations. In particular, the power law distributions of dislocation velocities [4] question the averaging procedure used in the Orowan's relation, as the variance of the distribution is ill-defined in this case (see above).

In this paper, we first review recent advances in the experimental characterization of slip heterogeneity and the associated spatial correlations of dislocation densities. We show 
The most direct method to investigate dislocation patterns is the analysis of transmission electron microscopy (TEM) images. Although TEM investigations of dislocation structures and patterns are countless, quantitative analyses of dislocation density spatial correlations as well as of statistical and scaling properties are difficult and tedious, and therefore scarce. As this subject was reviewed by Zaiser and Seeger [7], we simply recall here that the emergence of fractal, scale-free dislocation arrangements has been revealed by boxcounting measurements or by the distribution of dislocation cell sizes [11-13]. These fractal patterns are necessarily associated with long-ranged, non periodic spatial correlations, and differ in this respect to random arrangements (no correlations) or to patterns with periodic arrangements (periodic correlations). Periodic patterns seem to be a specific feature of cyclic deformation (fatigue)[7], a subject not treated here.

A complementary, but indirect method to explore the spatial heterogeneity of dislocation patterns is the $\mathrm{X}$-ray line profile analysis $[14,15]$, which allows to estimate the average dislocation density, $\langle\rho\rangle$, as well as the associated fluctuations quantified by the second-order moment of the distribution, $\left\langle\rho^{2}\right\rangle$. For compressed $\mathrm{Cu}$ single crystals, Szekely et al. [15] found a good (anti)correlation between the relative dislocation density fluctuation, quantified by the ratio $\delta=\left\langle\rho^{2}\right\rangle^{1 / 2} /\langle\rho\rangle$, and the fractal dimension of the dislocation patterns deduced from the distribution of cell sizes: the larger the heterogeneity, the larger the density fluctuation deduced from X-ray, and the smaller the fractal dimension deduced from TEM observations.

The most common method to study the spatial heterogeneity of slip and dislocation patterns, which was also the first developed, is the analysis of the surface of deformed samples. When dislocations emerge at the surface of a sample, slip steps are created. The heterogeneous nature of slip is then characterized by the development of roughness at the surface, which can be analyzed by shadowed microscopic images of sample surfaces or of 
replicas, or, more recently and directly, by atomic force microscopy (AFM) or scanning interferometry [16]. In his classic paper, Neuhauser [8] reviewed the observations available at that time, stressed the connection between the emergence of the so-called "slip bands" and collective dislocation motion, but did not perform any correlation or scaling analysis.

Sprusil and Hnilica [17] were the first to recognize the scale invariant, fractal character of slip patterns in plastically deformed Cd single crystals (figure 1), an hexagonal material in which the stage I of deformation is characterized by single-slip plasticity along the basal planes. Qualitatively, scale invariance is illustrated by the similarity of the patterns whatever the magnification. More quantitatively, these authors measured the number of dark lines, identified as "slip bands", per unit length along a line perpendicular to the basal planes, for each magnification, and obtained the following scaling (Figure 2):

$\rho_{b} \sim M^{1-D} \sim l^{D-1}$

where $\rho_{b}$ is the density of slip bands (in $\mathrm{m}^{-1}$ ) along a line perpendicular to the slip planes, $M$ the magnification, $l$ the image resolution $(l \sim 1 / M)$ and $D$ is the fractal dimension characterizing the set of slip band coordinates $(0<D<1)$. Sprusil and Hnilica reported a value of $D \approx 0.5$ for this set of points [17] (Figure 2). This fractal pattern fundamentally differs from a random, uncorrelated poissonian arrangement, and is an indication of long-ranged correlations. Using different methodologies (such as the so-called gap, yardstick or box-counting methods) applied on similar sets of slip band coordinates, Kleiser and Bocek [18] concluded on the scale invariant character of slip patterns in $\mathrm{Cu}$ single crystals, and reported $D$-values between 0.35 and 0.7 .

A strong limitation of these early works is that they did not resolve the elevation of the slip steps, which is related to the number of dislocations that glide along a given "slip band", or, in other words, to the local amount of plastic strain. Recent investigations based on AFM or scanning white-light interferometry allowed to quantitatively analyse this surface roughness of plastically deformed samples [16]. The elevation fluctuations (the roughness) can be linked to the spatial heterogeneity of strain, i.e. to local strain gradients. Therefore, by nature, the dislocation arrangements revealed by this technique are associated with strain gradients. The local elevation gradient (the local slope) $\partial z / \partial x$ is related to the local amount of plastic strain $\varepsilon_{p}(x)$ as follows [19]: 


$$
\frac{\partial z}{\partial x}=\varepsilon_{p}(x)-\left\langle\varepsilon_{p}\right\rangle
$$

where $z(x)$ is the elevation at position $x$ along the profile.

Nadgorny et al. [20] showed that an initially smooth surface of a $\mathrm{KCl}$ single crystal developed self-affine roughness during deformation. This self-affine scaling, documented on 1-d profiles of the surfaces, is expressed by:

$$
\langle|z(x)-z(x+\Delta x)|\rangle=\langle|\Delta z|\rangle \sim \Delta x^{H}
$$

where $\Delta x$ is the size of the window over which the elevation difference (a proxy of the roughness) is measured, and $H$ is the so-called Hurst's exponent characterizing the self-affine regime. Note that a more conventional estimate of roughness, the standard deviation of elevation over the window, is expected to follow the same scaling law [21]. For the stage I of deformation characterized by single-slip and low hardening, these authors reported a selfaffine scaling over the entire available range of observation (from about $300 \mathrm{~nm}$ to $200 \mu \mathrm{m}$ ) with $H \approx 0.65$ [20]. For stage II characterized by multi-slip and hardening, a small scale regime $(H \approx 0.7)$ seems to cross over to a large scale regime $(H \approx 0.5)$ above few tens of $\mu \mathrm{m}$. When the Hurst's exponents are larger than 0.5, this self-affine regime is the hallmark of scale invariance and long-ranged spatial correlations for the strain pattern, i.e. somehow for the dislocation density profile. Indeed, it implies that the power spectrum of the elevation profile $z(x)$ scales as (e.g. [22]):

$$
E(f) \sim f^{-\mu}(4)
$$

where $f$ is the frequency (in $\mathrm{m}^{-1}$ ), $E$ is the power spectrum, and the exponent $\mu$ is related to $H$ through $\mu=2 H+1$. Consequently, the gradient $\partial z / \partial x$, which is directly linked to the local plastic strain (see relation (2)), is also characterized by a power law spectrum, $E(f) \sim f^{-\alpha}$, where $\alpha=\mu$-2, i.e. $\alpha=0.3$ for $\mathrm{KCl}$ single crystals during stage I. As the power spectrum is the Fourier transform of the auto-correlation function [22], this power law behaviour with $\alpha>0$ is the hallmark of long-ranged spatial correlations of the plastic strain $\varepsilon_{p}(x)$. By comparison, an uncorrelated pattern would be characterized by a "white noise" with $\alpha=0$, i.e. $\mu=2$ and $H=0.5$ 
for the integrated profile. This might be the case for the strain pattern observed towards large scales during stage II $(H \approx 0.5$, see above).

In single crystals, at least during stage I, the upper bound of scaling is set by the sample size. Similar roughness analyses performed on polycrystalline $\mathrm{Cu}[16]$ an $\mathrm{Al}-\mathrm{Mg}$ alloys [23] revealed an auto-correlation function of the roughness profile $z(x)$ that saturates at scales larger than the average grain size $\langle d\rangle$. This defines $\langle d\rangle$ as the correlation length $\xi$ of the slip patterns. Towards small scales, scaling was observed down to the resolution scale of the analysis, i.e. down to $50 \mathrm{~nm}$ in the case of atomic force microscopy [16], with $0.8 \leq H \leq 0.92$, i.e. $0.6 \leq \alpha \leq 0.84$ for the power spectrum of the local strain pattern $\varepsilon_{p}(x)$.

The main drawback of these roughness analyses is that, as already stressed by Neuhauser [8], the presence of a free surface is expected to influence the operation of dislocation sources and dislocations patterns near the surface. Consequently, they might not be representative of bulk plasticity. Recently, we performed at ESRF post-mortem synchrotron X-ray topography analyses of ice single crystals deformed under pure torsion[24]. The torsion axis was parallel to the c-axis of the samples. Hexagonal ice Ih is characterized by single slip plasticity along the basal planes (i.e. stage II of deformation is never observed), which were therefore parallel to the shear plane in these experiments. Although a macroscopic strain gradient is geometrically imposed from the centre of the cylindrical samples to the surface, no gradient is imposed along the direction perpendicular to the basal planes. During synchrotron X-ray topography, the diffracted intensity records the distortion field, in our case related to orientation contrast. For our deformed samples, it is related to the dislocation density at the origin of the lattice distortion, i.e. only "excess dislocations" [25] responsible for lattice distortion are accessible by this technique. Diffracted intensity variations provide a map of dislocation density fluctuations within the bulk. Figure 3a shows an example of a diffraction pattern on the prismatic plane, with the long dimension corresponding to the height of the specimen, along the c-axis, while the width corresponds to the width of the specimen illuminated by the X-ray beam. Darker zones correspond to high dislocation density regions. The pattern shown on figure 3 a reveals a strong heterogeneity of the dislocation density along a direction perpendicular to the slip planes. We analyzed this heterogeneity along 1-d intensity profiles of the topograph perpendicular to the basal plane (figure 3b), by means of a spectral analysis. We observed a power law regime for the power spectrum of the intensity record over the entire available scale range (bounded by the resolution of the analysis, $10 \mu \mathrm{m}$, and the sample size, $\approx 7 \mathrm{~mm}$ ), $E(f) \sim f^{-\alpha}$, with $\alpha=1.3$ for all 
the samples analyzed [24](Figure 4). As for the elevation gradients $\partial z / \partial x$ of the surfaces of deformed samples (see above), these power laws reveal the scale invariance of the intensity records, i.e. of the dislocation density arrangement along a line perpendicular to the basal planes, as well as the associated long-range correlations. With this X-ray topography analysis, as the measured intensity is a proxy of the local dislocation density, the intensity gradients $\partial I / \partial x$ are a proxy of dislocation density gradients. The observed value of $\alpha=1.3<2$ identifies the intensity profile as a so-called anti-persistent pattern [26], i.e. a local increase of the density (positive gradient) is expected, in the probabilistic sense, to be surrounded by negative gradients. In other words, the density gradients are anti-correlated, although the absolute values of the gradients remain positively correlated[24].

Therefore, surface and bulk analyses of slip and dislocation patterns are both characterized by scale invariance and spatial long-range correlations, although the exponent $\alpha$ of the power spectrum is larger for the dislocation density patterns revealed by X-ray topography than those obtained for strain patterns from roughness analyses. In simpler words, bulk density patterns appear more correlated (less noisy) than surface strain patterns. At this stage, we don't know if this difference is due to the materials analyzed, to the difference between bulk and surface properties, or to the fact that a dislocation density cannot be directly translated into a local strain, even for the single-slip plasticity under torsion studied for the ice samples[24].

To end with this section about experimental characterization of slip patterns, one should mention that a 3-d analysis of dislocation avalanche locations monitored by acoustic emission during the creep of an ice single crystal revealed a scale-free pattern with a fractal dimension $D=2.5$, in agreement with the analyses detailed above [9].

\section{Slip bands, dislocation densities, strain-gradient plasticity and modelling frameworks}

The observations reported above argue for the rather ubiquitous character of spatial long-range correlations and scaling in dislocation and slip patterns. In single crystals, scaling holds from the smallest scales that can be explored by modern investigation methods (few tens of nm with AFM) to the scale of the sample. In polycrystalline materials, the correlation length $\xi$, i.e. the upper bound of scaling, seems to be set by the average grain size. 
These observations question the pertinence of classical and widely-used concepts such as slip bands or dislocation densities, and should constrain future modelling developments of plasticity.

\section{Slip bands and dislocations densities}

The concept of "slip band" emerged naturally when the spatial heterogeneity of plastic flow was recognized [8]. However, this a priori simple concept is misleading when scaling is present. Indeed, relation (1) implies that the density of slip bands $\rho_{b}$ is scale dependent: more and more slip bands per unit length appear as one increases the resolution. Any parameter related to $\rho_{b}$, such as, e.g., an average spacing between bands, has therefore to be taken with caution.

Similarly, the definition of a volumic statistical dislocation density $\rho$ implies that such averaging is meaningful at the scale considered, that is, the associated spatial fluctuations at that scale are small compared to the mean. In other words, the inevitable small scale heterogeneity (at the crystal lattice scale, dislocations are discrete defects) can be homogenised at some scale if the fluctuations vanish relatively to the mean above this scale. The scale invariance of the slip and dislocation patterns reported above might actually imply exactly the reverse. In the case of a so-called monofractal pattern, the ratio $\delta=\left\langle\rho^{2}\right\rangle^{1 / 2} /\langle\rho\rangle$ (as well as any higher order ratio $\left\langle\rho^{q}\right\rangle^{1 / q} /\langle\rho\rangle$ with $q>2$ ) remains constant whatever the scale of observation, i.e. fluctuations never vanish and dislocation density is ill-defined. For the dislocation patterns revealed by X-ray topography on ice samples, a detailed analysis might suggest a small departure from this monofractal scaling, with a possible decrease of $\delta$ with increasing scale as $\delta \sim \Delta x^{-\beta}$, with $\beta$ in the range 0.05-0.1 (figure 5). These estimated $\beta$-values are an upper bound, as $\delta$-values are biased (underestimated) by a finite size effect towards large scales. Therefore, this decrease of fluctuations towards large scale, if any, is very slow.

In the case of strain patterns revealed by surface roughness, Zaiser [19] checked on $\mathrm{KCl}$ single crystals that the surface roughness patterns were monofractal, i.e.:

$$
\left\langle|z(x)-z(x+\Delta x)|^{q}\right\rangle^{1 / q}=\left\langle|\Delta z|^{q}\right\rangle^{1 / q} \sim \Delta x^{H}, \forall q \text { for } 1 \leq q \leq 4
$$


, which shows that the strain fluctuations remain constant whatever the scale of observation, in good agreement with our analysis of dislocation density profiles.

This situation is true up to the correlation length $\xi$, meaning that a statistical dislocation density is ill-defined whatever the scale in the case of single crystal plasticity. On the other hand, in polycrystals, the average grain size $\langle d\rangle$ appears as an upper bound of scaling. Consequently, the fluctuations vanish as one coarsens the scale above $\langle d\rangle$. Mean-field approaches and homogenisation procedures might therefore be adequate if the representative volume element includes several grains (here, we do not consider other sources of heterogeneity such as textures and fabrics, which could be important at scales larger than $\langle d\rangle$ ). However, a recent analysis of dislocation avalanches in polycrystalline ice [27] showed that spatial correlations and fractal scaling extended towards scales much larger than the grain size. The role of grain boundaries as barriers to dislocation and slip correlations might not be so straightforward [28]. We note also that in single crystals, extended scaling was essentially observed during stage $\mathrm{I}$ (in ice and $\mathrm{KCl}$, see above), i.e. in a single slip configuration. During stage II in $\mathrm{KCl}$, a crossover towards a "white noise" regime $(H \approx 0.5)$ was observed at scales around few tens of $\mu \mathrm{m}$ [20]. This could indicate a rapid decrease of the dislocation density fluctuations above these scales, maybe as the result of forest-type interactions in different planes.

\section{Strain-gradient plasticity}

In order to account for a size dependence on strength in plasticity laws, the strain gradient theory introduced the concept of geometrically necessary dislocations (GND's), to be distinguished from the statistically stored dislocations (SSD's). From Ashby [29] the later are supposed to accumulate in pure crystal during straining and to be responsible for the forest hardening, while the first are required for the compatible deformation of various parts of the specimen. Ashby assumed that the deformation associated with the SSD's is spatially 
uniform, whereas GND's accommodate local strain-gradients, i.e. non-uniform deformation. In other words, the fluctuations of the SSD dislocation density, $\rho_{S}$, are assumed to vanish rapidly above the average spacing $l_{d}$. Within this framework, $\rho_{S}$ can be considered as a variable characterizing the state of deformation of the very material, while the GND density, $\rho_{G}$, is associated to "externally" imposed strain gradients. Here, we employ the term "external" with respect to the dislocation system itself, i.e. strain gradients imposed by microstructural features such as second-phase particles or grain boundaries, or imposed by the deformation geometry (e.g. bending tests). Such assumptions allowed to introduce a dependence on strain gradient, and then on a length scale, in phenomenological plasticity laws [30-32].

In this strain-gradient theory of plasticity, it is therefore assumed that the deformation of a single-phase single crystal will be spatially homogeneous and accommodated by SSD's only. This approximation about the existence of a plastically homogeneous deforming crystal can now be strongly questioned regarding the experimental results about dislocation patterning reported above. Surface measurements of dislocation induced slip steps in deformed single and polycrystals as well as X-ray diffraction analyses of dislocation density patterns revealed the emergence of strain (or dislocation density) gradients self-induced by the collective behaviour of dislocations. The dislocation and slip patterns observed in single crystals as well as in grains inside polycrystals are scale invariant, meaning that these selfinduced strain gradients exist whatever the scale of observation, within the limits of the scaling regime. The fact that fluctuations of dislocation density never vanish as one coarsen the scale (see previous section) implies that the concept of SSD's becomes meaningless, at least up to the upper bound of scaling. Note that the dislocation population which accommodates the externally imposed gradients, i.e. the so-called GND's in the Ashby's strain gradient plasticity theory, has, by nature, no long-range stresses associated with it [29]. On the other hand, the dislocation pattern which spontaneously emerge from collective effects is associated with long-range stresses, up to the correlation length $\xi$.

A geometrically necessary dislocation population can also be defined as the one accommodating the incompatibility of the lattice in the sense of the Nye's dislocation density tensor [33]. This tensor provides the net Burgers vector of all dislocation lines threading a given surface. This definition is more general than the original Ashby's definition of GND's [29]. Indeed, such dislocation population, also referred as "excess" dislocations [25], is, by nature, associated to long-ranged stresses and all kind of strain gradients, i.e. self-induced and 


\section{Modelling issues}

As perfectly illustrated by the contributions of this special issue, the density-based modelling of dislocation systems and plasticity is a very active and promising field of research. This might appear, at first glance, somehow in contradiction with the fact that statistical dislocation density is ill-defined (at least scale-dependent) when scaling is present (see above). However, we stress again that scaling is observed up to the correlation length which is set by the grain size in polycrystals. The case of single crystals, where scaling holds up to the sample size for the observations reported above, might appear as an "ideal" case without obvious practical application. On the other hand, the development of nanotechnologies, in shrinking the structural dimensions, could raise the problem of plastic heterogeneity in these cases.

Nevertheless, this emergence of scale-invariant patterns from the collective dislocation interactions necessarily challenges the modelling of plasticity. The key issue is, as stressed by Zaiser and Hochrainer [34], to achieve the transition between descriptions of plastic flow in terms of the dynamics of discrete dislocations (which have been successful in modelling the 
scale invariant properties of plasticity [4]), and continuum equations formulated in terms of partial differential equations and boundary value problems. The key challenge is to adapt the classical continuum modelling framework to take into account heterogeneity and fluctuations, while solving boundary value problems at the macro-scale.

A recent advance in this respect is based on the concept of "excess dislocations" briefly described in the previous section. It is an elegant way to propose a continuum framework that is able to (actually designed for) take into account internal stress fields and strain gradients resulting from dislocation interactions [35]. This framework, called Field Dislocation Mechanics (FDM) does not introduce phenomenological, ad hoc internal length scales to account for the observed gradients, instead incorporates physically-based length scales and spatial correlations due to dislocation interactions. In addition, FDM includes the dislocation transport equation [36], which is used as an evolution equation for the dislocation densities. It provides a dynamical setting for the solution of boundary value problems with stress and dislocation densities as state variables. When the resolution is small (of the order of the mean dislocation spacing), FDM is in practice only applicable to small size systems. However, coarse-graining is possible [25, 37]: short-range details of the internal stress field are smeared off but samples can be dealt with at a larger scale. It would be interesting in the future to test the ability of FDM to reproduce the complexity of self-induced strain gradients described in this paper.

Other recent developments (e.g. [38]) are based on mesoscopic field variables (stress, strain, dislocation densities). The phenomenological laws describing the evolution of these mesoscopic variables, such as dislocation density-based hardening laws, are inspired from the classical macroscopic modelling framework. Here, mesoscale means above the average dislocation spacing $l_{d}=1 / \rho^{1 / 2}$, such that field variables can be defined, but well below the macroscopic scale of the deforming body [19]. In addition to these phenomenological laws, some randomness is introduced in the model, e.g. on the local (mesoscopic) yield stress, to represent the sub-mesoscopic variability arising from the fine scale dynamics of interacting dislocations. Although this introduction of randomness is ad hoc, its statistical characteristics, such as variance or correlation properties, are deduced from experiments [15] or from discrete dislocation simulations. This association of (i) non-linear dynamics (a local yield stress), (ii) long-ranged elastic stresses, and (iii) randomness, is similar to statistical models of fracture of disordered media (e.g. [39, 40]) that successfully model the heterogeneity and scaling properties of fracture patterns, although in this last case the randomness introduced is uncorrelated (white noise). These stochastic models of plasticity successfully reproduce 


\section{Conclusion}

Recent detailed analyses of slip and dislocation density patterns in various deformed materials, either performed from a roughness analysis on the samples surface, or from X-ray topography within the bulk, revealed the emergence during deformation of scale invariant patterns characterized by long-range correlations, and resulting from the collective behaviour of dislocations. In single crystals, scale invariance holds up to the sample size, whereas in polycrystals, the upper bound of scaling, i.e. the correlation length, seems to be set by the average grain size. This scale invariance implies that the spatial fluctuations of dislocation density and/or slip never vanish as one coarsens the observation scale up to the correlation length. An immediate consequence is that a priori obvious concepts such as "slip bands" or dislocation density can be ill-defined. Similarly, the concept of "statistically stored dislocations" introduced by the strain gradient plasticity theory has to be taken with caution in these cases. Instead of the separation between GND's and SSD's as originally defined by Ashby [29], we propose to distinguish between "externally imposed" and "self-induced" strain gradients, and the associated dislocation populations. This scale invariant heterogeneity of slip emerging from the population of dislocations and their elastic mutual interactions also challenges the dislocation density-based modelling of plasticity. Recent modelling developments associating phenomenological laws on mesoscopic variables, long-ranged 
elastic stresses, and the introduction of randomness with statistical characteristics, such as variance or correlation properties, deduced from experiments or from discrete dislocation simulations, appear as promising directions.

Acknowledgments

We would like to thank M. Zaiser and C. Fressengeas for fruitful discussions, as well as Jurgen Hartwig and José Baruchel from the ID 19 beamline of ESRF. The comments of another anonymous reviewer are also acknowledged. 
1. Orowan, E., Zur Kristallplasticitat III. : Uber die Mechanismus des Gleitvorganges. Z. Physik, 1934. 89: p. 634-659.

2. Taylor, G.I., The Mechanism of Plastic Deformation of Crystals. Part I. Theoretical. Proc. Roy. Soc. A, 1934. 102: p. 362-387.

3. Sornette, D., Critical phenomena in natural sciences. Springer Series in Synergetics. 2000, Berlin: Springer.

4. Miguel, M.C., et al., Intermittent dislocation flow in viscoplastic deformation. Nature, 2001. 410: p. 667-671.

5. Taylor, G.I., Plastic strain in metals. J. Inst. Metals, 1938. 62: p. 307-324.

6. Kocks, U.F., Laws for work-hardening and low-temperature creep. J. Engng. Mater. Tech., 1976. 98: p. 76-85.

7. Zaiser, M. and A. Seeger, Long-range internal stresses, dislocation patterning and work-hardening in crystal plasticity, in Dislocations in solids, F.R.N. Nabarro and M.S. Duesbury, Editors. 2002, Elsevier. p. 1-100.

8. Neuhauser, H., Slip-line formation and collective dislocation motion, in Dislocations in solids, F.R.N. Nabarro, Editor. 1983, North-Holland Publishing Compagny. p. 319440.

9. Weiss, J. and D. Marsan, Three dimensional mapping of dislocation avalanches: clustering and space/time coupling. Science, 2003. 299(5603): p. 89-92.

10. Weiss, J. and M.-C. Miguel, Dislocation avalanche correlations. Mat. Sci. Eng. A, 2004. 387-389C(292-296).

11. Gil Sevillano, J., E. Bouchaud, and L.P. Kubin, The fractal nature of gliding dislocation lines. Scripta Met. Mat., 1991. 25: p. 355-360.

12. Gil Sevillano, J., Patterns in heavily deformed metals. Physica Scripta, 1993. T49: p. 405 .

13. Hahner, P., K. Bay, and M. Zaiser, Fractal dislocation patterning during plastic deformation. Phys. Rev. Lett., 1998. 81: p. 2470-2473.

14. Ungar, T., Dislocation densities, arrangements and character from X-ray diffraction experiments. Mat. Sci. Eng., 2001. 309-310: p. 14-22.

15. Szekely, F., I. Groma, and J. Lendvai, Statistic properties of dislocation structures investigated by X-ray diffraction. Mat. Sci. Eng. A, 2001. 309-310: p. 352-355. 
16. Zaiser, M., et al., Self-affine surface morphology of plastically deformed metals. Phys. Rev. Lett., 2004. 93(19): p. 195507.

17. Sprusil, B. and F. Hnilica, Fractal character of slip lines of Cd single crystals. Czech. J. Phys., 1985. 35: p. 897-900.

18. Kleiser, T. and M. Bocek, The fractal nature of slip in crystals. Z. Metallkde, 1986. 77: p. 582-587.

19. Zaiser, M., Scale invariance in plastic flow of crystalline solids. Advances in Physics, 2006. 55: p. 185-245.

20. Nadgorny, E., et al. Evolution of self-affine surface roughness in plastically deforming KCl single crystals. in SMPRI2005. 2005: PoS.

21. Schmittbuhl, J., J.P. Vilotte, and S. Roux, Reliability of self-affine measurements. Physical Review E, 1995. 51: p. 131-147.

22. Schmittbuhl, J., F. Schmitt, and C. Scholz, Scaling invariance of crack surfaces. J. Geophys. Res., 1995. 100: p. 5953-5973.

23. Wouters, O., et al., On the evolution of surface roughness during deformation of polycrystalline aluminium alloys. Acta Mat., 2005. 53: p. 4043-4050.

24. Montagnat, M., et al., The heterogeneous nature of slip in ice single crystals deformed under torsion. Philos. Mag. A, 2006. in press.

25. Varadhan, S., A.J. Beaudoin, and C. Fressengeas. Coupling the dynamics of statistically distributed and excess dislocations. in International Conference on Statistical Mechanics of Plasticity and Related Instabilities. 2005. Bangalore, India.

26. Mandelbrot, B. and J. Van Ness, Fractional Brownian motions, fractional noises and applications. SIAM Review, 1968. 10(4): p. 422-437.

27. Richeton, T., J. Weiss, and F. Louchet, Dislocation avalanches: role of temperature, grain size and strain hardening. Acta Materiala, 2005. 53: p. 4463-4471.

28. Richeton, T., J. Weiss, and F. Louchet, Breakdown of avalanche critical behaviour in polycrystalline plasticity. Nature Materials, 2005. 4: p. 465-469.

29. Ashby, M.F., The deformation of plastically non-homogeneous materials. Philos. Mag., 1970. 13: p. 399-424.

30. Fleck, N.A., et al., Strain gradient plasticity: theory and experiments. Acta Metall. Mater., 1994. 42: p. 475-487.

31. Fleck, N.A. and J.W. Hutchinson, Strain gradient plasticity. J. Mech. Phys. Solids, 1997. 41: p. 1825. 
32. Gao, H., Y. Huang, and W.D. Nix, Modeling plasticity at the micrometer scale. Naturwissenschaften, 1999. 86: p. 507-515.

33. Nye, J.F., Some geometrical relations in dislocated crystals. Acta. Metall., 1953. 1: p. 153.

34. Zaiser, M. and T. Hochrainer, Some steps towards a continuum representation of $3 D$ dislocation systems. Scripta Materiala, 2006. 54: p. 717-721.

35. Acharya, A., A model of crystal plasticity based on the theory of continuously distributed dislocations. J. Mech. Phys. Solids, 2001. 49: p. 761-784.

36. Mura, T., Continuous distribution of moving dislocations. Phil. Mag., 1963. 89: p. 843.

37. Archarya, A. and A. Roy, Size effects and idealized dislocation microstructure at small scales: Predictions of a phenomenological model of mesoscopic field dislocation mechanics: Part I. J. Mech. Phys. Solids, 2006. 54: p. 1687-1710.

38. Zaiser, M. and P. Moretti, Fluctuation phenomena in crystal plasticity - a continuum model. J. Stat. Mech., 2005: p. P08004.

39. Herrmann, H.J. and S. Roux, Statistical models for the fracture of disordered media. 1990, Amsterdam: North-Holland.

40. Cowie, P.A., D. Sornette, and C. Vanneste, Multifractal scaling properties of a growing fault population. Geophys. J. Int., 1995. 122: p. 457-469. 


\section{Figure captions:}

Figure 1. Slip patterns observed on the surface of plastically deformed Cd single crystals at different magnifications $M$. (a) $M=100$, (b) $M=200$, (c) $M=1000$, (d) $M=2000$, (e) $M=10000$, (f) $M=20000$ (from [17])

Figure 2. Evolution of the number of "slip bands" $N$, for a given fixed length on the photographs, with magnification $M$ for the slip patterns shown on figure 1. (from [17]). The density of "slip bands" is given by $\rho_{b}=N \times M$

Figure 3. a) Synchrotron X-ray topograph obtained on a slice of an ice single crystal deformed under pure torsion (c-axis, and then torsion direction is represented). b) Intensity (arbitrary unit) profile obtained along this topograph.

Figure 4. Fourier power spectrum of the intensity record of figure $3 b$. The slope $\alpha=1.3$ is represented. The white dots are averages over bin regularly separated in logarithmic scale.

Figure 5. Evolution of the spatial fluctuations of the dislocation density $\rho$, measured by the ratio $\delta$, with the scale of observation, for the intensity profile of figure $3 \mathrm{~b}$. The intensity $I$ is a proxy of $\rho$, so $\delta$ is determined from $\delta=\left\langle\rho^{2}\right\rangle_{\Delta x}{ }^{1 / 2} /\langle\rho\rangle_{\Delta x}$ 

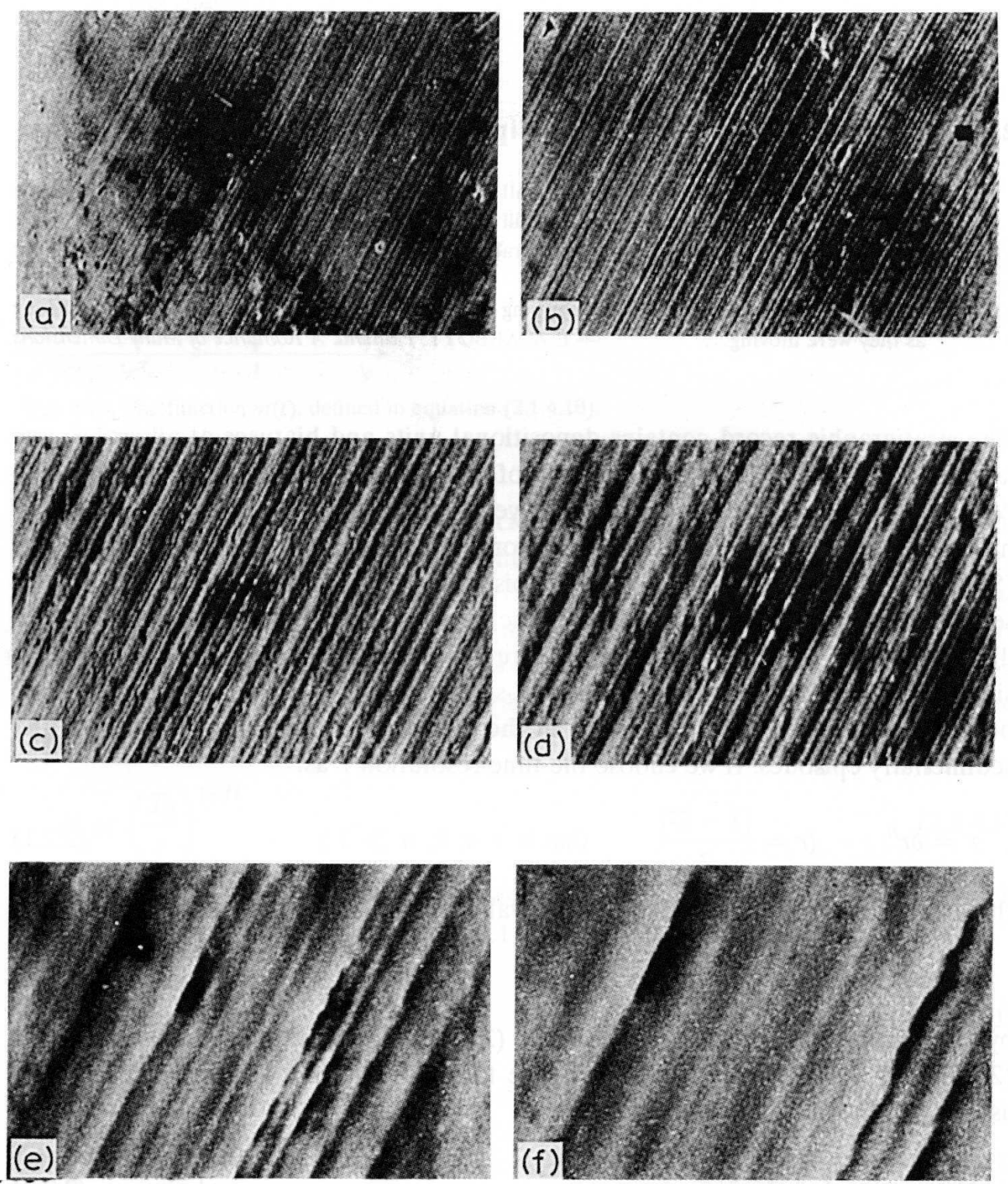

Figure 1

$126 \times 150 \mathrm{~mm}(300 \times 300 \mathrm{DPI})$ 


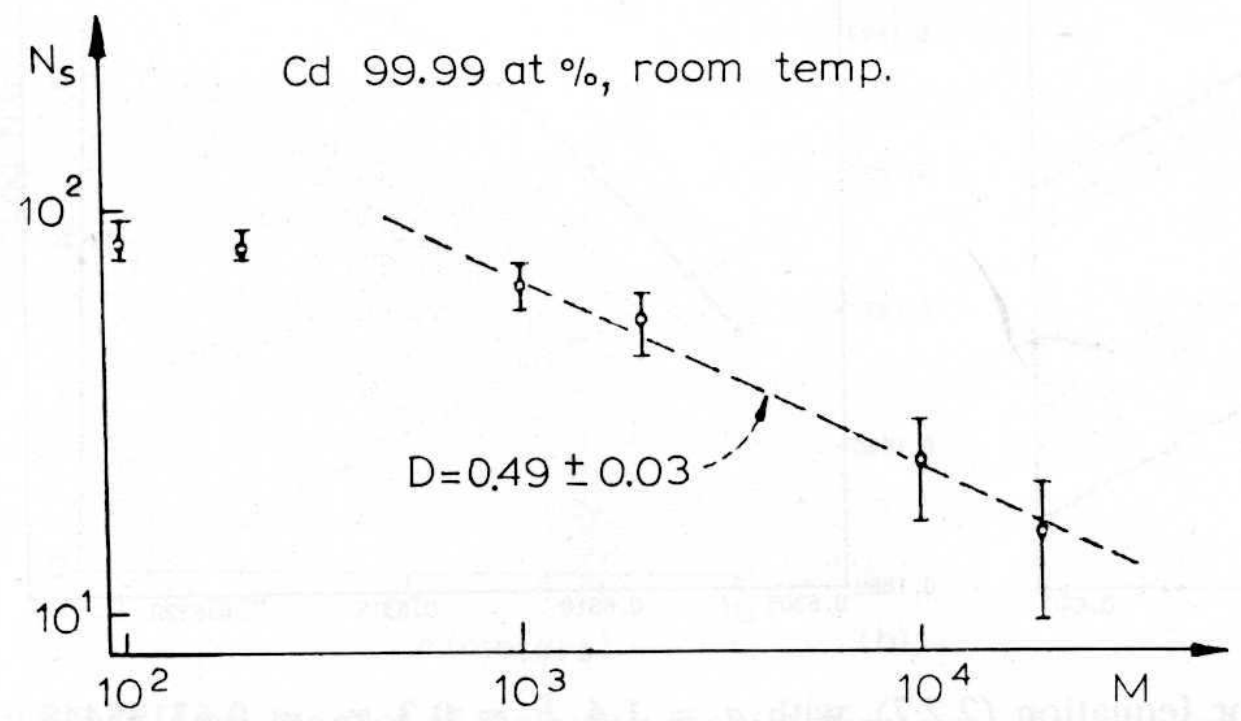

Figure 2

$84 \times 52 \mathrm{~mm}(300 \times 300$ DPI $)$ 

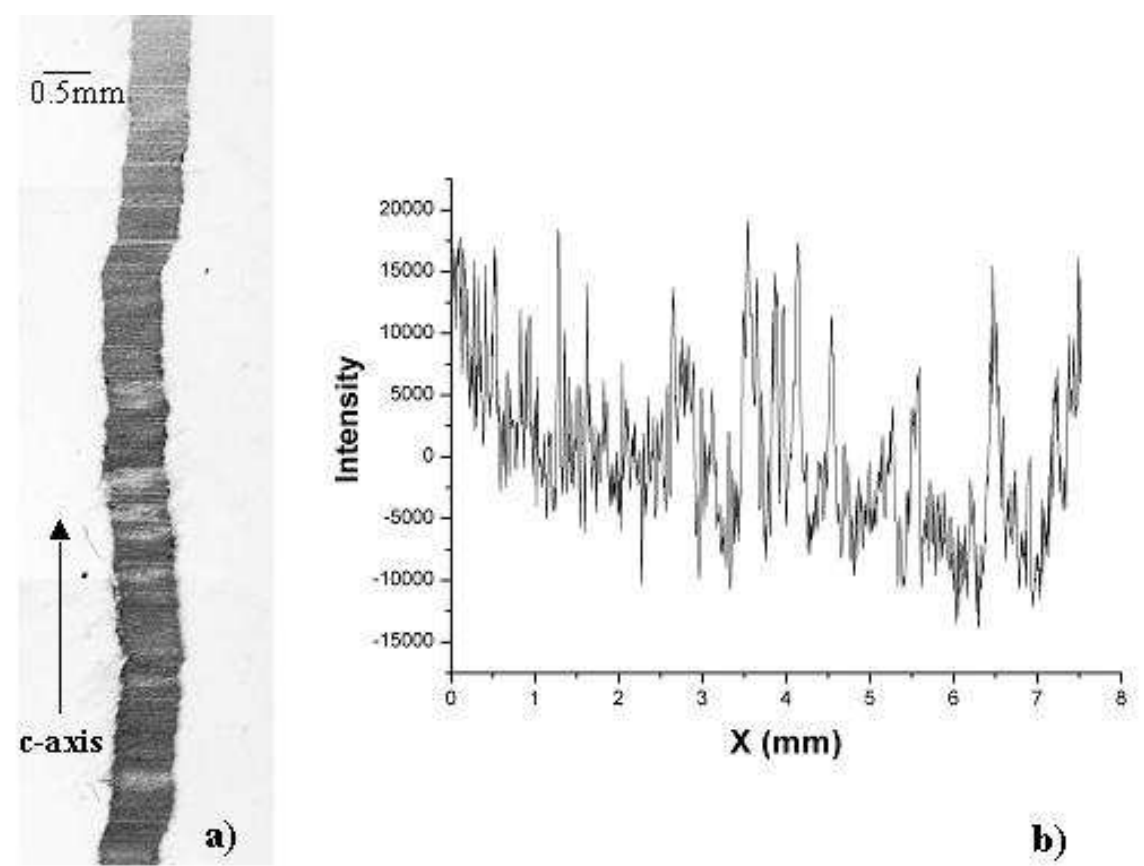

a)

b)

Figure 3

$48 \times 36 \mathrm{~mm}(300 \times 300 \mathrm{DPI})$

35

36

37

38

39

40

41

42

43

44

45

46

47

48

49

50

51

52

53

54

55

56

57

58

59

60

http://mc.manuscriptcentral.com/pm-pml 


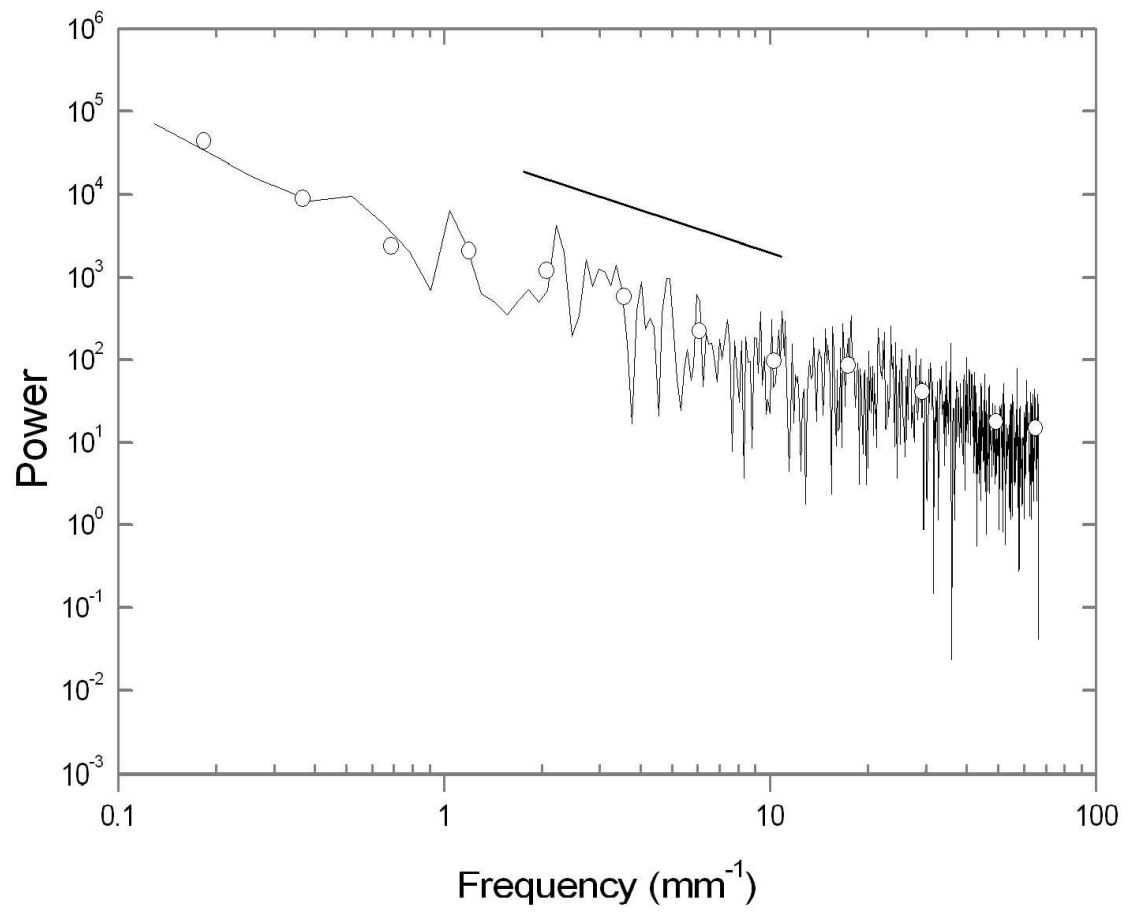

Figure 4

$279 \times 215 \mathrm{~mm}(150 \times 150 \mathrm{DPI})$

http://mc.manuscriptcentral.com/pm-pml 
